Revista de Comunicación y Salud, 2018, Vol. 8, № 1, pp. 99-109 Editado por Cátedra de Comunicación y Salud

ISSN: 2173-1675

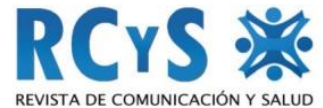

Enviado 19 de septiembre de 2018

Aprobado 31 de octubre de 2018

\title{
ANÁLISIS DE IMPACTOS DE LOS MEDIOS DE COMUNICACIÓN DE LOS CASOS DE LA FIEBRE HEMORRÁGICA DE CRIMEA-CONGO EN LA COMUNIDAD DE MADRID (2016). EL PAPEL DE LA COMUNICACIÓN INSTITUCIONAL EN CRISIS DE SALUD PÚBLICA
}

\author{
Analysis of media impacts on cases of Crimean-Congo haemorrhagic fever in the \\ Community of Madrid (2016). The role of media in public health crisis \\ Pilar Mestre Ortega ${ }^{1}$, Juan José Bote Ruiz-de Gordoa, Amparo Mira Roldán, Rocío \\ Buendía Domínguez y Miguel Ángel Bravo Hernández \\ Consejería de Sanidad, Comunidad de Madrid
}

\section{Resumen}

El 31 de agosto de 2016 se notifican dos casos de sospecha de la fiebre hemorrágica de Crimea- Congo al Servicio de Epidemiologia de la Comunidad de Madrid. Una de las personas afectadas ha fallecido. Se trata de una enfermedad desconocida en nuestro ámbito, contagiosa y de la que no existen precedentes en la Europa Occidental. De manera inmediata, el Departamento de Comunicación de la Consejería de Sanidad de la Comunidad de Madrid activa un protocolo de comunicación para informar sobre la evolución de la investigación epidemiológica, preservando la identidad de los pacientes y profesionales afectados por el caso e insistiendo en trasladar un mensaje de tranquilidad a la población. Se identifica un portavoz técnico para los medios de comunicación. A la vez se incluye la información en la página web de la Comunidad de Madrid, dirigida al ciudadano en general y toda la información sanitaria en la Intranet corporativa para que estén debidamente informados los profesionales sanitarios. De los 36 días en que estuvo activada la alerta, de los impactos evaluados (676) en los 42 medios de comunicación analizados (radio, prensa, televisión, digitales y medios especializados), tan solo se produjo una información negativa. La alerta se desactivó el 6 de octubre sin que se hubiese producido durante la misma alarma social.

Palabras clave: comunicación institucional, departamentos de comunicación, impactos de medios de comunicación, crisis de salud pública, fiebre hemorrágica de CrimeaCongo.

\footnotetext{
${ }^{1}$ Autor para correspondencia: Pilar Mestre Ortega pilar.mestre@salud.madrid.org
} 
Análisis de impactos de los medios de comunicación de los casos de la fiebre hemorrágica de Crimea-Congo en la Comunidad de Madrid (2016)...

\begin{abstract}
On August 31, 2016, two suspected cases of Crimean-Congo haemorrhagic fever (CCHF) were reported to the Epidemiology Service of the Community of Madrid. One of the persons affected has died in the meantime. CCHF is a contagious disease and without precedents in Western Europe. The Press Office of the Community of Madrid's Health Department immediately activated a communication protocol to inform on the evolution of epidemiological research, preserving the identity of the patients and professionals and insisting on transferring a message of tranquillity to the population. A technical spokesperson was available to satisfy the demands of the media. Information aimed at the general public was included on the website of the Community of Madrid. All health information was made available on the corporate intranet so that health professionals were duly informed. The alert was activated during 36 days and only one negative information was produced regarding the evaluated impacts (676) in the 42 media analysed (radio, press, television, digital and specialized media). The alert was deactivated on October 6 without social alarm having occurred.
\end{abstract}

Keywords: institutional communication, department of communication, public health crisis, media impacts, Crimean-Congo hemorrhagic fever.

\title{
Cómo citar el artículo
}

Mestre Ortega, P.; Bote Ruiz-de Gordoa, J. J.; Mira Roldán, A.; Buendía Domínguez, R. y Bravo Hernández, M. A. (2018). Análisis de impactos de los medios de comunicación de los casos de la fiebre hemorrágica de Crimea-Congo en la Comunidad de Madrid (2018). El papel de la comunicación institucional en crisis de salud pública. Revista de Comunicación y Salud, 8(1), pp. 99-109.

DOI: http://doi.org/10.35669/revistadecomunicacionysalud.2018.8(1).99-109

\section{INTRODUCCIÓN}

El miércoles 31 de agosto de 2016 la Dirección General de Salud Pública de la Comunidad de Madrid informa en sus canales internos de la Consejería de Sanidad de dos casos de sospecha de fiebre hemorrágica de Crimea-Congo. Una de las personas con sospecha de esta enfermedad ha fallecido. Se trata de una enfermedad desconocida en nuestro país, contagiosa y de la que no existen precedentes en la Europa Occidental.

La persona fallecida, un hombre de 62 años, pudo haber contraído la enfermedad por la picadura de una garrapata mientras paseaba por un pueblo de Ávila. La segunda persona afectada es una enfermera del Hospital Universitario Infanta Leonor que atendió al primero que se encuentra ingresada con pronóstico grave en la Unidad de Aislamiento de Alto Nivel del Hospital Universitario La Paz-Carlos III de Madrid. Al día siguiente, se confirma por Laboratorio la enfermedad.

En el momento en que recibe la información, el Departamento de Comunicación de la Consejería de Sanidad de la Comunidad de Madrid activa un protocolo de 
Análisis de impactos de los medios de comunicación de los casos de la fiebre hemorrágica de Crimea-Congo en la Comunidad de Madrid (2016)...

comunicación para informar a los medios de comunicación sobre la evolución de la investigación, de una manera sistemática: a la misma hora - salvo cambios en la investigación-, con un portavoz técnico, ofreciendo la misma información mediante comunicado (que se envía por correo electrónico) a todos, en igualdad de contenidos y condiciones y preservando la identidad de los pacientes y profesionales afectados por el caso.

Durante este período, las actuaciones de la Dirección General de Salud Pública de la Comunidad de Madrid se centraron en la recuperación de la sanitaria afectada, en realizar seguimiento médico a 437 personas (Negredo, 2017: 159) que podrían haber estado en contacto con el virus (sanitarios, familiares...), adoptar medidas preventivas, como así ocurrió con la hospitalización de tres personas con síntomas de la enfermedad en unidades de aislamiento y, por último en proteger a los 59 profesionales que atendieron directa o indirectamente a la enfermera en la Unidad de Aislamiento de Alto Nivel del Hospital Universitario La Paz-Carlos III.

El objetivo general de esta investigación consiste en analizar la repercusión de la alerta de salud pública en los medios de comunicación para determinar si se generó alarma social, por un lado, y si los medios de comunicación siguieron la estrategia informativa y actuaron de aliados o no en la transmisión de la información en una situación excepcional.

\section{MARCO TEÓRICO}

Las crisis o alertas de salud pública afectan fundamentalmente a dos ámbitos, ligados en muchas ocasiones. Por un lado, implican una repercusión sanitaria con impacto en la salud de la población y en las medidas que la institución pone en marcha; en segunda instancia, hay que considerar el espacio de la percepción social, en cómo los ciudadanos interpreten el problema y su reacción ante el mismo. De esta manera, la Dirección General de Salud Pública de la Comunidad de Madrid define una alerta de salud pública como "la sospecha de una situación de potencial riesgo para la salud de la población y/o de trascendencia social frente a la cual sea necesario el desarrollo de acciones de salud pública urgentes y eficaces". (Dirección General de Salud Pública de la Comunidad de Madrid: www.madrid.org).

La multiplicidad de factores que acompañan a una situación de alerta o crisis de salud pública puede en algunos casos restar valor a la eficacia de acciones de comunicación, pero indiscutiblemente los autores en este campo sostienen que es clave contar con protocolos de comunicación, como abunda Martínez Segura (2006, p. 446447) insistiendo en que pese a que en muchas ocasiones no se pueden controlar debido, entre otras consideraciones, por la percepción de la población, la mejor prevención consiste en estar preparado: "la protocolización de los pasos a seguir, son las mejores herramientas para que una vez producido el incendio haya una serie de profesionales y expertos que sepan que es lo que se debe hacer."

Revista de Comunicación y Salud, 2018, Vol. 8, no 1, pp. 99-109 
Análisis de impactos de los medios de comunicación de los casos de la fiebre hemorrágica de Crimea-Congo en la Comunidad de Madrid (2016)...

En todo caso, cada crisis de salud pública debe contar con una planificación según sus características. Entre las diez medidas que enumera March (2011, pp. 61-66) sobre la gestión de la crisis una es contar con una estrategia de medios de información.

Existen elementos de consenso en la gestión de comunicación en una alerta o crisis de salud pública. La Organización Mundial de la Salud destaca cuatro principios que deben regir la información de brotes epidémicos: rapidez de respuesta, transparencia, planificación, y en primera instancia confianza, sin la cual la ciudadanía no creerá "en la información sanitaria que las autoridades nacionales transmitan o bien, no obrarán en consecuencia" (OMS, 2018, p. 5).

Las experiencias en la gestión de crisis sanitarias, como redunda García Frontinán (2017, p. 452) avalan el papel de la comunicación como un elemento clave que en el caso de la gripe A se sostuvo en un "un contacto constante con los periodistas para demostrar transparencia y lograr la confianza de la población en las instituciones y evitar, de este modo, posibles situaciones de caos y descontrol social".

Las relaciones con los medios de comunicación deben generarse antes de las crisis, es decir, son fruto de un trabajo anterior y diario del Departamento de Comunicación en su día a día con la difusión de los mensajes de la organización sanitaria, que va a generar un conocimiento de los medios, de las necesidades de los periodistas. Así Sanjuán (2007, p. 80) incide en que se exige una planificación previa que "debería incluir una política de relación permanente con los medios y no sólo cuando tengamos algo que vender o cuando ellos nos atosiguen por su interés en algo".

La transparencia de la gestión de la organización se refuerza con portavoces cualificados y técnicos, subraya Revuelta (2016, p. 7). "El portavoz ideal sería una persona que tuviera a la vez experiencia y autoridad". Por su parte Rodríguez (2017, p. 374 ) insiste en la importancia de portavoces técnicos "con mayor credibilidad entre el público, que contribuyan a reforzar el mensaje de la organización".

En este sentido, la "transparencia" también debe estar en armonía con la confidencialidad de los pacientes y el respeto a su imagen, como se puso de manifiesto con la Gripe A y García Frontiñán (2017, p. 454) recuerda que los datos clínicos son confidenciales "y propiedad de los propios pacientes por lo que los únicos que podrían facilitárselos a los medios son ellos mismos".

La coordinación interna de la institución debe ir a la vez de la mano de una coordinación interinstitucional de todos los niveles, incluyendo los departamentos de prensa, para evitar contradicciones. Autores como García Frontiñán (2017, p. 452) destacan el acierto de la coordinación interinstitucional como en la crisis de la gripe A. "Los medios de comunicación, responsables políticos y comunicadores institucionales tanto del ámbito nacional, como regional y local, estuvieron en contacto directo de manera continua". 
Análisis de impactos de los medios de comunicación de los casos de la fiebre hemorrágica de Crimea-Congo en la Comunidad de Madrid (2016)...

El derecho a la información implica también que los diferentes responsables en la gestión de una epidemia deben colaborar con los medios de comunicación. Del mismo modo, los medios de comunicación tienen el deber de informar sin poner en peligro la salud pública o la buena marcha de las actuaciones que se estén llevando a cabo para la gestión y control de la epidemia, destaca en sus conclusiones el Informe Quiral 2014 (2014, p. 55).

\section{ANÁLISIS DE IMPACTOS}

En este trabajo se ha realizado un análisis de los impactos en los medios de comunicación recogidos por el Departamento de Comunicación de la Consejería de Sanidad durante los 36 días en que estuvo activada la alerta: del 31 de agosto al 6 de octubre de 2016 y también se recoge el día posterior (07) el que se registran impactos informando del fin de la alerta. En total se ha analizado la información aparecida en 42 medios de comunicación: 20 medios audiovisuales y 22 medios de comunicación de prensa escrita, digital o agencias.

En total, se han recopilado 676 impactos, de los que 387 se localizan en medios audiovisuales (radio y televisión) y el resto, 285 , en prensa, digitales y agencia:

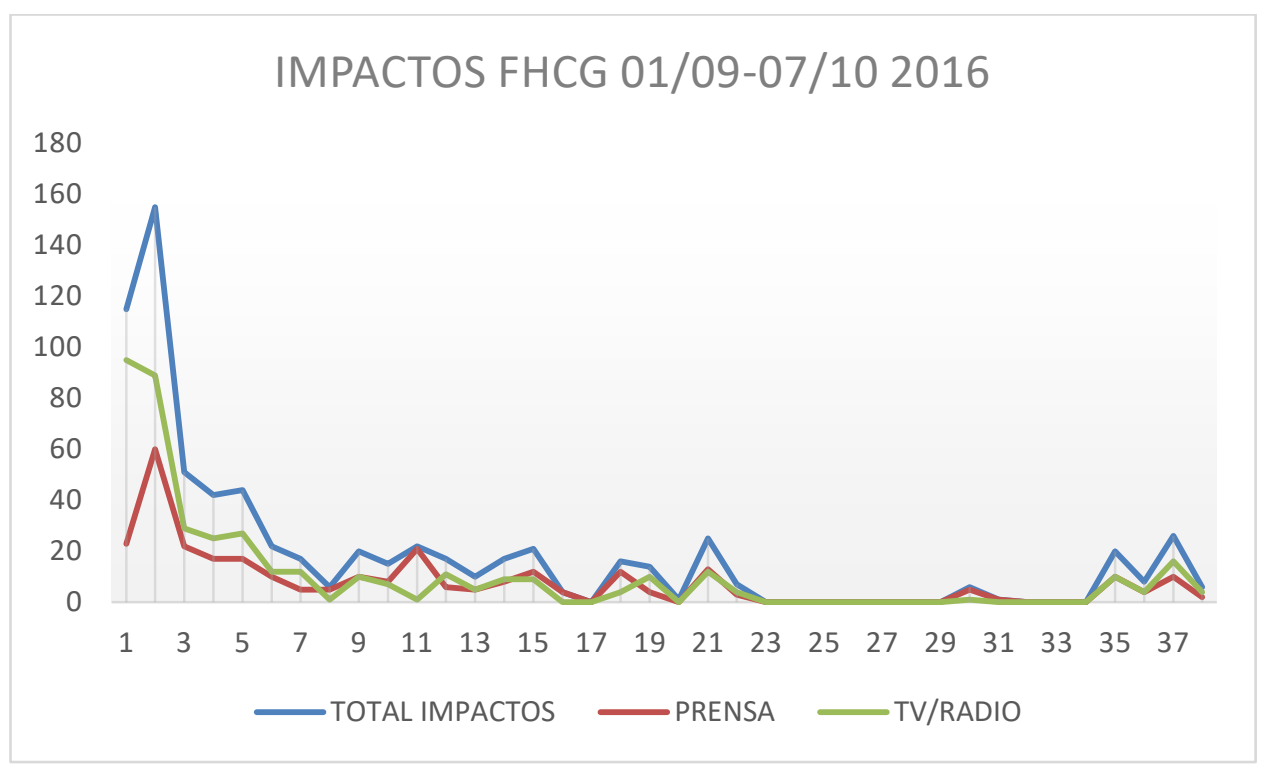

\section{Grafico1.}

Día 1: 1 de septiembre. Activación de alerta Día: 37: 6 de octubre. Comunicación Fin de Alerta

Cabe destacar que se produjeron tres picos de información: el máximo se centra en los dos primeros días de la alerta (1 y 2 de septiembre) cuando se anuncia la activación del protocolo y la rueda de prensa del día siguiente, que recoge casi la mitad de los 
Análisis de impactos de los medios de comunicación de los casos de la fiebre hemorrágica de Crimea-Congo en la Comunidad de Madrid (2016)...

registros periodísticos compilados (267) con predominio de los medios audiovisuales (184).

Se genera un siguiente aumento de difusión de la información institucional cuando se comunica que la paciente está libre del virus y su posterior alta médica el 21 de septiembre (con 25 impactos) pero muy alejado del interés que despertó el inicio de la alerta (18 y 21 de septiembre); y por último, cuando se anuncia el final del seguimiento de todos los contactos y se da por cerrada la alerta sanitaria (6 y 7 de octubre con 32 registros periodísticos).

De estos 36 días, la única información negativa referente a la gestión del Departamento de Comunicación de la Consejería de Sanidad en el caso se publicó en el medio digital eldiario.es que editó una noticia bajo el título: Sanidad activó el protocolo contra el virus de la garrapata 11 días tarde, con la enfermera ya contagiada Este extremo fue desmentido por el Departamento de Comunicación de la Consejería explicando con datos contrastados que el protocolo se activó en cuanto se conoció que se trataba de un caso de fiebre hemorrágica de Crimea-Congo.

En este sentido el ECDC (2018:6) destaca que la mayor parte de la cobertura de los medios se realizó en la primera semana después del diagnóstico inicial de FHCC "y fue, en su mayor parte, objetiva y precisa, lo que refleja las actualizaciones periódicas de información recibidas de las autoridades". El organismo europeo pone, a la vez de relieve la información que se facilitó a la comunidad para que se mantuviera "alerta pero no alarmada y la mayoría de las personas siguieron este consejo...solo se registraron 41 llamadas en una línea directa durante un período de 31 días, lo que indica un bajo nivel de preocupación en la población en general".

Otro elemento que pudo distorsionar la gestión de la comunicación fue la noticia publicada también por eldiario.es sobre los antecedentes de la existencia del virus y las medidas de prevención en esta crisis, al recoger la noticia titulada "Un estudio ya alertó de que el virus de Crimea-Congo estaba reemergiendo en España", del jueves 1 de septiembre y en la que se explicaba que un grupo de expertos, a instancias del Ministerio de Sanidad, "realizaron un informe advirtiendo de la posibilidad de la llegada a España del virus".

Esta noticia apenas tuvo trascendencia a nivel general, aunque tuvo repercusión en la Cadena Ser (viernes 2 de septiembre), y contó con un pronunciamiento del Colegio de Médicos sin mayor recorrido: "Aunque las autoridades insisten en transmitir un mensaje de tranquilidad respecto a la fiebre hemorrágica de Crimea-Congo detectada en Madrid, algunos expertos, entre ellos el Colegio de Médicos de Madrid, reclaman que el Ministerio de Sanidad actualice los datos del informe que se publicó en 2011".

Precisamente las sociedades científicas y sindicatos representativos de los profesionales sí se pronunciaron sobre la oportunidad de los protocolos, sin que llegara a generarse polémica y además con puntos de vista diferentes. 
Análisis de impactos de los medios de comunicación de los casos de la fiebre hemorrágica de Crimea-Congo en la Comunidad de Madrid (2016)...

Es el caso del sindicato de Enfermería SATSE, cuya opinión recoge el viernes 2 de septiembre Onda Cero que "destaca el incumplimiento de los protocolos previstos ante patologías contagiosas".

De manera contraria se posiciona el sindicato de Sanidad CSIF, en Onda Cero (viernes 2 de septiembre) "La enfermera afectada de fiebre hemorrágica, contagiada por el paciente que murió en agosto, evoluciona favorablemente. Sanidad sigue pendiente de la evolución de las 190 personas que estuvieron en contacto con ambos. El portavoz de Sanidad de CSIF, pide mantener el protocolo".

Otras posiciones de la profesión sanitaria en Onda Madrid (viernes 2 de septiembre) expresan tibieza en el asunto: "La enfermera infectada por la fiebre hemorrágica Crimea Congo sigue estable dentro de la gravedad, en el Carlos III. Los colectivos de enfermería y medicina destacan la rápida actuación de los profesionales y demandan una actualización de los protocolos. De igual manera Redacción Médica (el viernes 2 de septiembre): Los colegios profesionales piden información y valoran el trabajo de los profesionales".

Tan solo se registró una información errónea al difundir la Agencia EFE el uno de octubre (cinco días antes de que oficialmente se diera por finalizada la alerta) un teletipo en el que daba por cerrada la alerta y que emitieron por el cómputo que el periodista hizo incompleto del período de seguimiento de los contactos de los enfermos ya que no tuvo en cuenta a otros profesionales no asistenciales (limpieza, Laboratorio.). El País se hizo eco de la noticia en su edición impresa.

El análisis de los portavoces oficiales durante los dos primeros días de la gestión pone de manifiesto la relevancia de su papel (con más de 20 impactos en los dos primeros). El portavoz Ángel Gil concedió entrevistas (del uno al 5 de septiembre) a Telemadrid Noticias 1(01.09.16), Onda Cero (01.09.16); Telemadrid Contigo (02.09.16) El Rompeolas de Onda Madrid (02.09.16), Telemadrid Noticias (02.09.16), en Más Vale Tarde de la Sexta (02.09.16), y en Telemadrid Diario de la Noche (03.09.016). Además, fue el portavoz de la rueda de prensa convocada de manera urgente el sábado 3 de septiembre para explicar nuevos ingresos, de la que se hicieron eco EFE, TVE-1, TVG, Antena 3, Telemadrid, la Sexta, Abc, El País, La Razón, El Periódico, Cadena Ser, Tele 5, Onda Cero, confidencial.com, madridactual,com, entre otros medios.

El jueves 6 de octubre, la Comunidad de Madrid informa de que se da por zanjada la alerta de la fiebre hemorrágica Crimea-Congo al cumplirse el fin del seguimiento médico de todos los profesionales sanitarios y no sanitarios que habían estado en contacto con la paciente directa o indirectamente.

Entre otras informaciones que se hicieron eco de la gestión de la comunicación durante esta alerta se encuentra el programa La Ventana de Madrid de la Cadena Ser (el 21 de septiembre a las 19:30h), el día que la presidenta de la Comunidad anunció en un acto que se celebraba en el Hospital Universitario Puerta de Hierro-Majadahonda que se daba el alta a la enfermera contagiada por el virus. El periodista cerró la 
Análisis de impactos de los medios de comunicación de los casos de la fiebre hemorrágica de Crimea-Congo en la Comunidad de Madrid (2016)...

información indicando "por ser justos, la gestión de esta crisis ha sido impecable por parte de la Consejería de Sanidad".

Por su parte, la agencia de noticias PRNoticias emitió una información el viernes 2 de septiembre en la que analizaba la gestión de la comunicación repasando los elementos claves en la gestión: "Los expertos en comunicación de crisis detallan que los puntos que se deben tener en cuenta son: ofrecer toda la información disponible con transparencia y veracidad, designar portavoces expertos y adecuados, evitar rumores y mensajes contradictorios que generen alarmismo, respetar la intimidad de los afectados, de su familia y de su entorno, e informar con puntualidad, transparencia y datos actualizados. Con esta estrategia la Consejería de Sanidad se ha adelantado a cualquier filtración de información, ha evitado la proliferación de bulos y ha conseguido que la administración no volviese a ir a remolque de las noticias que aparecen en los medios de Comunicación, como ocurrió en la gestión de la crisis del ébola".

\section{CONCLUSIONES}

Los casos de FHCC de la Comunidad de Madrid generaron una gran demanda informativa tanto a nivel local como nacional (e internacional, aunque no se ha computado) con un registro de 676 impactos durante el período en que se mantuvo activada la alerta sin que se generara alarma social. Tan solo, y al principio de la alerta, se registró una información cuestionando la gestión de la crisis que fue rápidamente contestada por el Departamento de Comunicación. La gran atención de los medios de comunicación se centró en los dos primeros días de activación de alerta (jueves 1 y viernes 2 de septiembre), que acaparan casi la mitad de los registros. Y por lo tanto, los primeros días fueron determinantes para fijar la orientación, mensajes y posicionamiento frente a la alerta de los medios de comunicación.

La estrategia comunicativa de los casos de FHCC se llevó a cabo sin que se generara alarma social ni grandes titulares, imágenes sensacionalistas 0 cuestionamientos por parte de los medios de comunicación sobre las medidas adoptadas.

Especialmente al principio de la alerta, los portavoces oficiales técnicos de la Consejería de Sanidad se convirtieron en una ayuda sólida para los medios de comunicación que reclamaban fuentes oficiales para aclarar dudas o contar con testimonios directos de la investigación, con gran accesibilidad para los periodistas. Esta iniciativa ha sido reconocida por el ECDC (2018:7) como una buena práctica para promover colaboraciones y sinergias en los problemas de salud pública transfronterizos y desarrollar un protocolo, que es establecer un comité de crisis e identificar a un portavoz.

\section{BIBLIOGRAFÍA}

Dirección General de Salud Pública de la Comunidad de Madrid (n.d.). Sistema de Alerta Rápida en Salud Pública. 
Análisis de impactos de los medios de comunicación de los casos de la fiebre hemorrágica de Crimea-Congo en la Comunidad de Madrid (2016)...

Recuperado de

http://www.madrid.org/cs/Satellite?blobcol=urldata\&blobheader=application\%2Fpdf\&b lobheadername1=Content-

disposition\&blobheadername2=cadena\&blobheadervalue1=filename\%3DTriptico Ale rtasSP.pdf.pdf\&blobheadervalue2=language\%3Des\%26site\%3DPortalSalud\&blobkey $=$ id\&blobtable=MungoBlobs\&blobwhere $=1352926997772 \&$ ssbinary=true

European Centre For Disease Prevention and Control (ECDC). (2018). Case study: Enablers and Barriers for Community and Institutional Public Health Emergency Preparedness Synergies. Crimean-Congo Hemorrhagic Fever in Spain. ECDC. (Documento inédito, recibido el 5 de marzo de 2018).

Fiebre hemorrágica, ¿por qué no se ha convertido en una crisis de comunicación como la del ébola? Septiembre 2, 2016. PRNoticias. Recuperado de https://prnoticias.com/salud/periodismo-y-comunicacion-en-salud/20155590-fiebrehemorragica-y-crisis-de-comunicacion.

García Frontiñán, E. (2017). Comunicación de crisis en los gabinetes de prensa sanitarios. Análisis de la producción periodística y de la información publicada sobre la gripe $A$. (Tesis Doctoral). Universidad Complutense de Madrid. Recuperado de http://cisne.sim.ucm.es/record=b3698962 S6*spi\#tabs.

Informe Quiral 2014. (2014). La comunicación pública sobre la enfermedad del Ébola. Fundación Privada Vila Casas. Observatorio de la Comunicación Científica. Recuperado de http://www.fundaciovilacasas.com/es/informe-quiral

March, J. C. (2012). El riesgo de una mala comunicación de riesgos. Revista de Comunicación y Salud, 1(2), 61-66. Recuperado de http://revistadecomunicacionysalud.org/index.php/rcys/article.

Martínez Segura, P. (2006). Crisis sanitarias y medios de comunicación. Revista de Administración Sanitaria, 4(3), 437-438

Negredo A, de la Calle-Prieto F, Palencia-Herrejón E, et al. (2017). Autochthonous Crimean-Congo hemorrhagic fever in Spain (2017). New England Journal of Medicine, 377, 154-61. DOI: 10.1056/NEJMoa1615162

Organización Mundial de la Salud (OMS) (2018). Guía de la Organización Mundial de la Salud para planificar la comunicación en caso de brotes epidémicos. Recuperado de http://apps.who.int/iris/bitstream/10665/44162/1/9789243597447 spa.pdf

Revuelta G y Morales, Pol (Coords.) (2016). Debate sobre periodismo científico. El tratamiento informativo del brote epidémico del virus del Ébola. Cuadernos de la Fundación Dr. Antonio Esteve, 2(40). Recuperado de http://www.esteve. org/?wpdmact=process\&did=MTU00C5ob3RsaW5r

Rodríguez Andrés, R. (2017). Cómo diseñar planes de comunicación para organizaciones sanitarias (pp. 367-378). En U. Cuesta, C. Peñafiel, J. L. Terrón, E. Bustamante, S. Gaspar. (Coords.) Manual de Comunicación y Salud. Madrid: Dextra Editorial.

Sanjuán, A. (2007). El método del caso aplicado a las estrategias informativas en situación de crisis. En M. Túñez, (Coord) y A. Sanjuán. Comunicación. Preventiva, planificación y ejecución de estrategias de información interna y externa ante situaciones de crisis (pp. 73-88). Netbilblo.com. Recuperado de http://hdl.handle.net/2183/11904 
Análisis de impactos de los medios de comunicación de los casos de la fiebre hemorrágica de Crimea-Congo en la Comunidad de Madrid (2016)...

Otras referencias:

- Información sobre la fiebre hemorrágica de Crimea-Congo. Disponible en www.madrid.org y Portal Salud.

- Documentación del Departamento de Comunicación de la Consejería de Sanidad. Análisis de impactos de la FHCC (31 de agosto-7 de octubre de 2016)

- Documentos internos del Departamento de Comunicación de la Consejería de Sanidad (septiembre de 2016).

Noticias sobre FHCB del 31 de agosto a 6 de octubre 2016, recopiladas en:

\section{TELEVISIONES Y RADIO}

- TVE

- Antena 3

- La Sexta

- Cuatro TV

- Tele 5

- Telemadrid TV

- TVG

- $T V 3$

- $E T B$

- Canal Sur TV

- Canal Sur Radio

- Es Radio

- Cadena Ser

- Onda Cero

- Radio Galega

- Cadena Cope

- Canal Sur Radio

- Catalunya Radio

- RNE

- Onda Madrid

\section{PRENSA ESCRITA/DIGITALES Y AGENCIAS}

- $E F E$

- Europa Press

- Servimedia

- El País

- El Mundo

- $A B C$

- La Razón

- La Vanguardia 
Análisis de impactos de los medios de comunicación de los casos de la fiebre hemorrágica de Crimea-Congo en la Comunidad de Madrid (2016)...

- El Periódico

- 20 Minutos

- eldiario.es

- redaccionmedica.com

- consalud.es

- madridiario.es

- madridactual.es

- vozpopuli.com

- estrelladigital.es

- eleconomista.es

- elplural.com

- elconfidencial.com

- huffingtonpost.es

- libertaddigital.com 